\title{
Study on the Corneal Higher-Order Aberrations and Correlation in Patients with Different Degrees of Myopia Suitable for Wavefront-Guided FS-LASIK
}

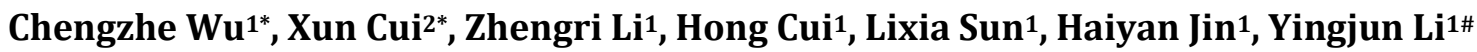 \\ ${ }^{1}$ Department of Ophthalmology, Affiliated Hospital of Yanbian University, Yanji, China \\ ${ }^{2}$ Department of Physiology and Pathophysiology, School of Medical Sciences, Yanbian University, Yanji, China \\ Email:"liyingjun1301@163.com
}

How to cite this paper: $\mathrm{Wu}, \mathrm{C} . Z$., Cui, X., Li, Z.R., Cui, H., Sun, L.X., Jin, H.Y. and Li, Y.J. (2020) Study on the Corneal HigherOrder Aberrations and Correlation in $\mathrm{Pa}$ tients with Different Degrees of Myopia Suitable for Wavefront-Guided FS-LASIK. Journal of Biosciences and Medicines, 8 , 13-22.

https://doi.org/10.4236/jbm.2020.81003

Received: October 10, 2019

Accepted: December 23, 2019

Published: December 26, 2019

Copyright $\odot 2020$ by author(s) and Scientific Research Publishing Inc. This work is licensed under the Creative Commons Attribution International License (CC BY 4.0).

http://creativecommons.org/licenses/by/4.0/

\section{(c) (i) Open Access}

\begin{abstract}
Background: Individualized corneal refractive surgery requires an understanding of the basis of higher-order aberrations before surgery. To investigate the characteristics and distribution of anterior surface wavefront aberrations in patients suitable for corneal refractive surgery. Methods: A total of 121 myopic patients (121 eyes, 18 - 45 years old) who underwent corneal refractive surgery were recruited from January to May 2016 at Affiliated Hospital, Yanbian University. Patients were randomly selected by the Pentacam anterior segment analysis system with a spherical equivalent (SE) of -0.25 to $-10.00 \mathrm{D}$. The corneal anterior aberrations (total higher-order aberration; spherical aberration; Coma; Trefoil) and $\mathrm{Q}$ and $\mathrm{K}$ values were detected, and a correlation analysis of the relevant parameters was carried out. Results: The root-mean-square (RMS) of the third to sixth order aberrations of the corneal anterior wavefront aberrations at a $6 \mathrm{~mm}$ analysis diameter showed a decreasing trend in patients suitable for the corneal refractive surgery, and the RMS of the third order aberrations accounted for $62.92 \%$ of the total HOAs. The coma ratio (coma/total cornea higher-order aberrations) was increased with the increasing diopters, while the spherical aberration ratio (spherical aberration/total cornea higher-order aberrations) was not changed. In addition, the spherical aberration was $0.203 \pm 0.082 \mu \mathrm{m}$ (range: 0.061 to 0.503 $\mu \mathrm{m}$ ), and the $\mathrm{Q}_{30}$ was $-0.19 \pm 0.03$ (range: -0.58 to 0.31 ). There were significant differences in the coma aberrations of preoperative corneal anterior surface $(3,1)$ between the low, middle and high myopia groups $(P=0.013)$. The spherical equivalent was positively correlated with the corneal coma of the anterior corneal surfaces $(R=-0.241, P=0.009)$, and the $\mathrm{Q}$ value was positively
\end{abstract}

${ }^{\star}$ Chengzhe $\mathrm{Wu}$ and Xun Cui contributed equally to this research. 
correlated with the total higher-order aberrations $(R=0.326, P<0.001)$. Conclusions: Individual wavefront aberrations on the anterior surfaces of the cornea are comparatively different, and the Zernike coefficients are related to the degree of myopia. Spherical aberrations are the most overriding aberrations of the cornea.

\section{Keywords}

Corneal Refractive Surgery, Anterior Corneal Surface, Higher-Order Aberration, Spherical Aberration

\section{Introduction}

Wavefront aberration is the deviation between the actual and the ideal wavefront, including wavefront aberrations of the cornea and inner eye, which are the main factors affecting visual quality. The connotation of wavefront aberrations can be expressed by the Zernike polynomial periodic table [1].

The wavefront is divided into low and higher-order aberrations. Myopia, hypermetropia and astigmatism are low order wave front aberrations, while spherical and coma aberrations are higher-order wave front aberrations. Conventional corneal laser refractive surgery significantly improves higher-order aberrations and corrects low order aberrations, and consequently significantly improves visual quality [2] [3] [4]. Precise measurement of preoperative HOAs is essential to maximize the effects of wavefront-guided custom ablation. Although there have been numerous studies on wavefront aberrations in the integral eye, there are few studies on wavefront aberrations of the cornea.

Corneal refractive surgery is performed on the anterior surface of the cornea. The increase in wavefront aberrations after femtosecond laser-assisted laser in situ keratomileusis (FS-LASIK) is mainly due to changes in wavefront aberrations on the anterior corneal surface because changes on the posterior surface of the cornea are smaller [4]. The aim of our study is to investigate the wavefront aberrations on the anterior surface of the cornea in a myopic population and to provide a clinical basis for designing a more reasonable wavefront-guided individualized corneal refractive surgery.

\section{Materials and Methods}

\subsection{Patients}

From January 2016 to May 2016, 121 patients underwent FS-LASIK surgery (Department of Ophthalmology, the Affiliated Hospital of Yanbian University) to correct myopia, including 59 males and 62 females, aged 18 - 45 years with an average of $25.58 \pm 5.90$ years old. This study evaluated healthy emmetropic eyes with an best corrected visual acuity of $20 / 20$ or better, The patients had spherical equivalent power ranging from -025 to -10.00 diopters (D); astigmatisms $\leq 1.50$ 
$\mathrm{D}$; intraocular pressure ranging from 8.5 to $20.1 \mathrm{mmHg}$, average $12.49 \pm 2.85$ $\mathrm{mmHg}$; and corneal thinnest point thickness ranging from 493 to $621 \mu \mathrm{m}$, average $524.96 \pm 26.67 \mu \mathrm{m}$. Low myopia $(<-3.00 \mathrm{D})$, moderate myopia $(-3.00 \mathrm{D}$ to $-6.00 \mathrm{D})$ and high myopia (>-6.00 D).

The patients were required to wear corneal contact lenses for more than 2 weeks. All eyes had normal binocular function and a relatively large interpupillary distance $(>6.0 \mathrm{~cm})$. Exclusion criteria included ocular pathology, ophthalmic disorders, amblyopia, strabismus, previous intraocular surgery, laser treatment, and retinal complications. Each patient was enrolled in only the right eye for study. The institutional review board at the Affiliated Hospital of Yanbian University approved this study, which followed the tenets of the Declaration of Helsinki. Written informed consent was obtained from all patients after the nature and possible consequences of the study were explained.

\subsection{Wavefront Aberration Measurement}

The Pentacam system (Oculus, Inc, USA) was used to measure the higher-order aberrations on the corneal anterior surface with a $6.0-\mathrm{mm}$ area at the center of the cornea. During the test, the patients were required to take a seat and stare at the fixation target, a blue band at the center of the rotation axis in the Pent cam system. Aiming and focusing in the system were achieved by an ophthalmologist skilled in the operation of the system using a joystick according to the instructions on the screen and $180^{\circ}$ scanning of Scheimpflug 3D imaging performed within $2 \mathrm{~s}$. Each patient was tested by the same ophthalmologist. The measurement was taken 3 times consecutively for trefoil, spherical, and coma aberrations. The average values were calculated for trefoil, spherical, and coma aberrations. The average values of the root-mean-square higher-order aberrations (RMS HOAs) for trefoil aberration were calculated.

\subsection{Operation Groups}

The patients were divided into 3 groups by myopic degree: the low group included 42 patients with 42 eyes, the moderate group included 40 patients with 40 eyes, and the high group contained 39 patients with 39 eyes.

\section{Statistics}

The continuous variables were summarized by means \pm standard deviations (SDs) and ranges. The categorical variables were summarized by frequencies and proportions. Analysis of variance (ANOVA) along with Duncan's multiple range test was used to compare wavefront variables among the three groups. A simple linear regression model and Spearman's rank correlation coefficient were used to assess the relationship between nuclear lens density and internal optics aberration. A $P$ value of less than 0.05 was considered statistically significant. All data analysis was performed using SPSS version 12 (SPSS, Inc., Chicago, IL, USA). 


\section{Results}

A total of 121 patients (59 men and 62 women) were evaluated. The mean age of the patients was $25.58 \pm 5.90$ years (range 18 to 45 years). The average corneal curvature was $42.62 \pm 1.23 \mathrm{D}$ (range 39.32 to $48.91 \mathrm{D}$ ). In addition, the mean values for the spherical equivalent (SE), axial length $(\mathrm{mm})$, sphere $(\mathrm{D})$ and cylinder (D) are shown in Table 1. Differences in the binocular sphere degree were $\leq 2.50 \mathrm{D}$.

The root-mean-square (RMS) of (the third to the sixth order) aberrations of the corneal higher-order wave surface aberrations at a $6 \mathrm{~mm}$ analysis diameter in different myopia groups are shown in Table 2. As shown in Table 2, the Zernike coefficients progressively decrease the RMS values from the third to the sixth order, and the total higher-order aberrations at an RMS of the third order aberrations was approximately $62.96 \%$. There were some changes in spherical aberrations and coma aberrations before corneal refractive surgery: the spherical aberrations accounted for $53.3 \%, 52.1 \%$ and $42.5 \%$, in the low, moderate and high myopia groups, respectively, and the coma aberrations were $42.2 \%, 56.4 \%$ and $62.3 \%$, respectively. At a $6 \mathrm{~mm}$ analysis diameter, the total corneal higher-order aberration was $0.351 \pm 0.152 \mu \mathrm{m}$, and the spherical aberration was $0.203 \pm 0.082$ $\mu \mathrm{m}$ (range from 0.061 to $0.503 \mu \mathrm{m}$ ), in which $71.6 \%, 19.2 \%$ and $32.7 \%$ of the patient eyes were at $0.15-0.30 \mu \mathrm{m}, 0.25-0.30 \mu \mathrm{m}$ and $0.20-0.25 \mu \mathrm{m}$, respectively.

In addition, the coma and trefoil aberrations were $0.134 \pm 0.052 \mu \mathrm{m}$ and 0.157 $\pm 0.094 \mu \mathrm{m}$, respectively. The aberrations of the corneal anterior surface between the low, moderate and high myopia groups $(3,1)$ were significantly decreased $(P$ $=0.013$ ) (Figure 1). Moreover, as the diopter increased, the coma aberration ratio (coma aberration/total cornea higher-order aberrations) increased, but the while the spherical aberration not changed in the cornea (Figure 2).

Table 1. Characteristics of patients and surgical data.

\begin{tabular}{ccc}
\hline & Mean \pm SD & Range \\
\hline Age (years) & $25.58 \pm 5.90$ & 18 to 45 \\
SE (D) & $-5.74 \pm 1.91$ & 0 to -10.50 \\
Axial length (mm) & $26.51 \pm 1.49$ & 23.09 to 31.85 \\
Sphere (D) & $-4.93 \pm 1.75$ & -0.25 to -9.63 \\
Cylinder (D) & $-0.79 \pm 0.81$ & 0 to -2.00 \\
\hline
\end{tabular}

Table 2. Mean levels of RMS ( $\mu \mathrm{m})$ in different myopic groups.

\begin{tabular}{cccccc}
\hline Refractive error & $\mathrm{n}$ & Total HOA & Coma & SA & Trefoil \\
\hline High myopes (-10.00 to -6.00 DS) & 39 & $0.47 \pm 0.12$ & $0.23 \pm 0.11$ & $0.10 \pm 0.06$ & $0.24 \pm 0.12$ \\
Moderate myopes (-6.00 to -3.00 DS) & 40 & $0.43 \pm 0.15$ & $0.23 \pm 0.12$ & $0.11 \pm 0.07$ & $0.22 \pm 0.13$ \\
Low myopes (-3.00 to -0.75 DS) & 42 & $0.45 \pm 0.11$ & $0.19 \pm 0.08$ & $0.14 \pm 0.10$ & $0.17 \pm 0.08$ \\
$P$ & & 0.154 & 0.016 & 0.203 & 0.541 \\
\hline
\end{tabular}

RMS HOAs, root-mean-square of higher-order aberrations; SA, spherical aberration. 


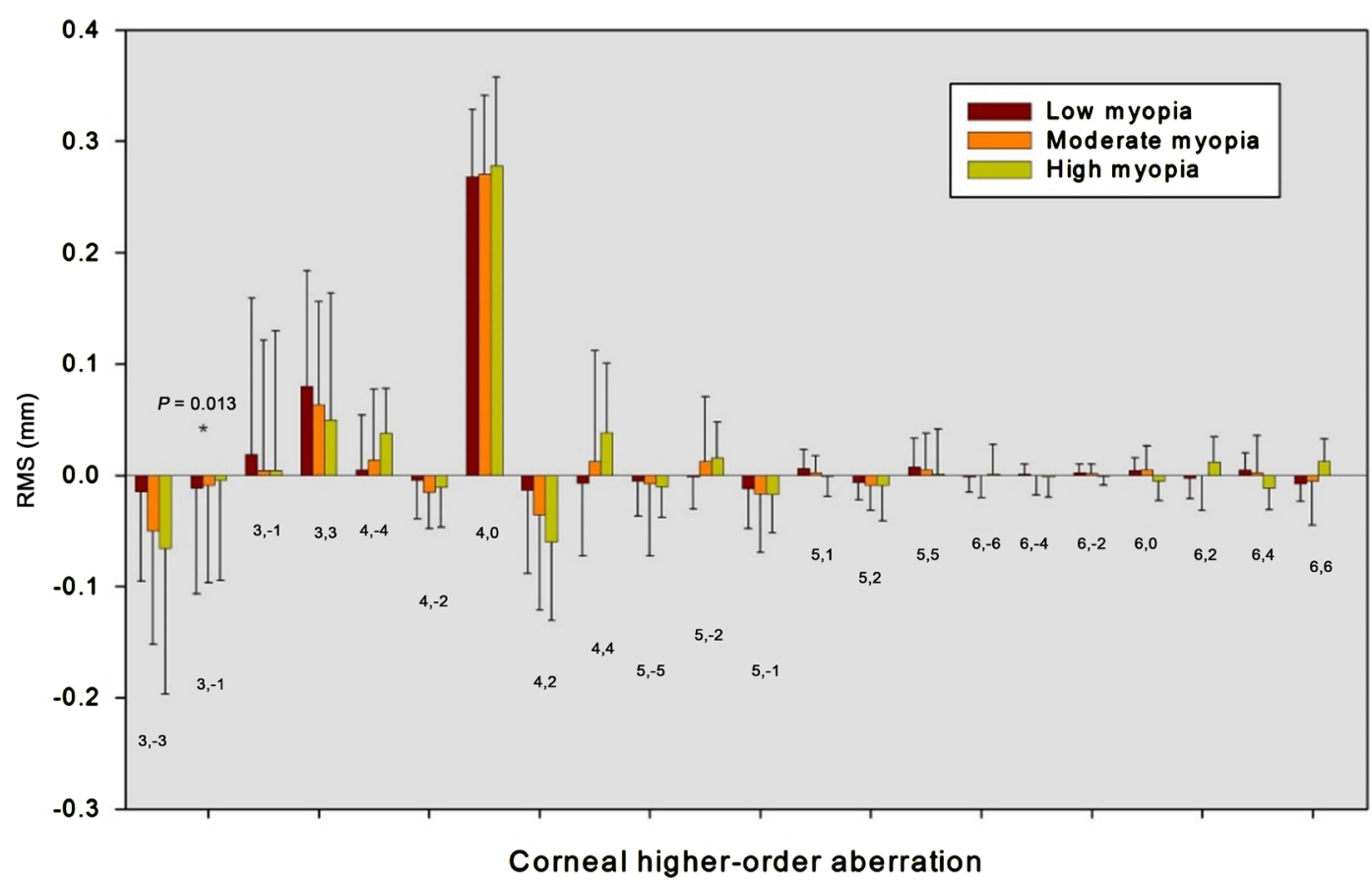

Figure 1. The anterior corneal aberrations of different myopia groups were compared.
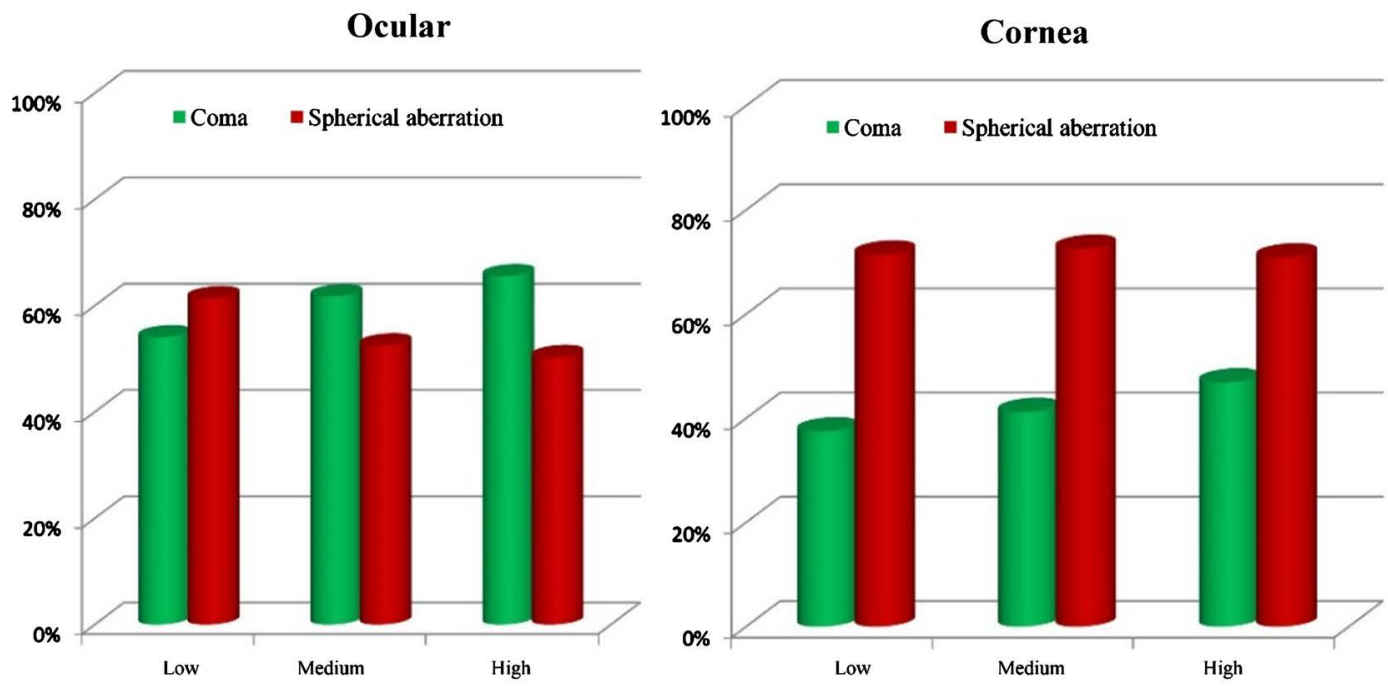

Figure 2. The ratios of coma and spherical aberration in different myopia groups.

The Q30 value at the $6 \mathrm{~mm}$ analysis diameter of the anterior corneal surface suitable for excimer laser surgery in patients was $-0.19 \pm 0.03$ (range: -0.58 to 0.31). The numbers of eyes with negative Q20, Q25, Q30, Q35 and Q40 values accounted for $81.21 \%, 87.31 \%, 90.14 \%, 100 \%$ and $93.24 \%$ of the total eyes, respectively (Figure 3 ).

In addition, the results of the correlation analysis showed that the spherical equivalent power was positively correlated with the coma of the anterior corneal surfaces $(R=-0.241, P=0.009)$, but there was no correlation with the total higher-order, spherical or trefoil aberrations $(R=0.073, P=0.435 ; R=0.039, P$ 
$=0.671 ; R=0.027, P=0.770$, respectively, Figure 4). Furthermore, the $\mathrm{Q}$ value was positively correlated with the total higher-order aberrations $(R=0.326, P<$ $0.001)$, but was not correlate with coma or trefoil aberrations $(R=0.103, P=$ 0.535 and $R=0.065, P=0.977$, respectively, Figure 5).

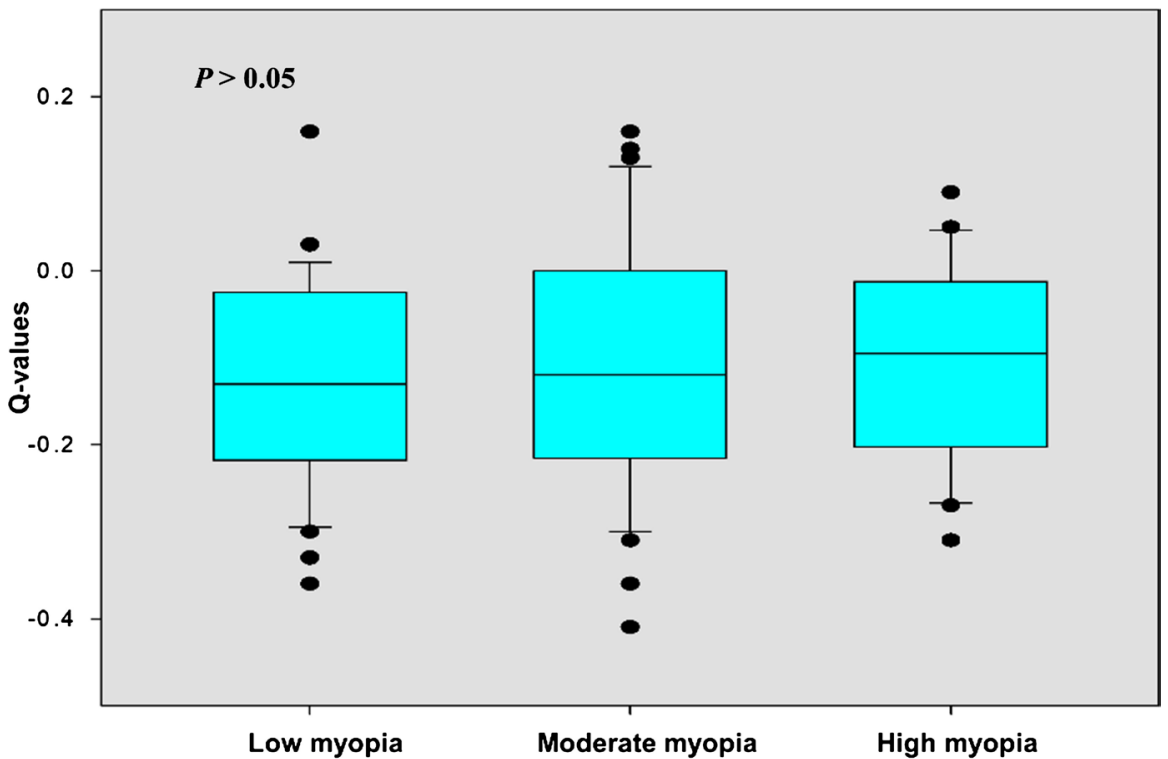

Figure 3. The Q values of different myopia groups were compared.
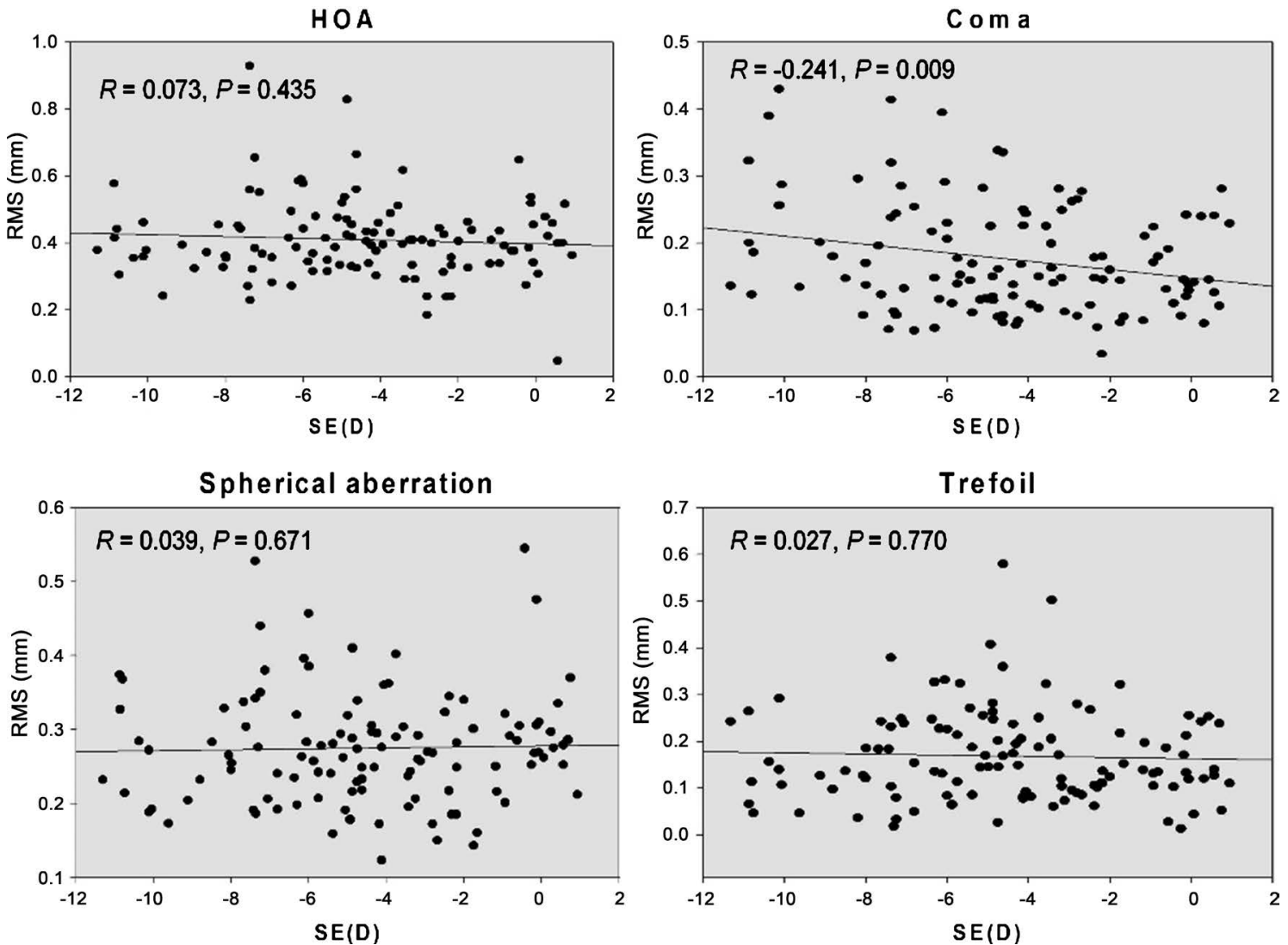

Figure 4. Correlations between spherical equivalents and anterior corneal aberrations. 

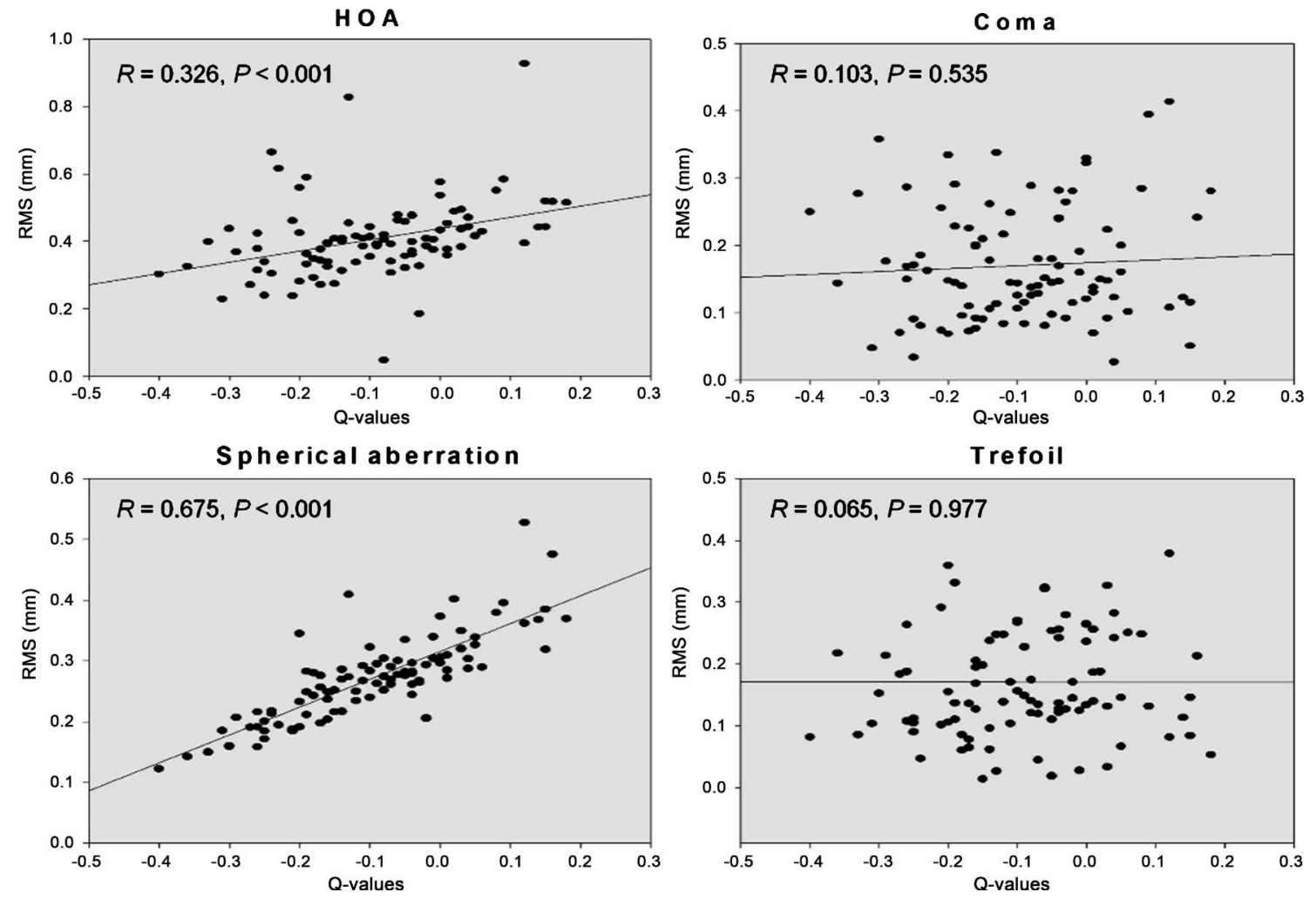

Figure 5. Correlations between the $\mathrm{Q}$ value and anterior corneal aberrations.

\section{Discussion}

With the continuous development of corneal refractive surgery and the further updating of related equipment, excimer laser corneal refractive surgery has become a procedure with high safety, predictability and rapid recovery. Based on the worldwide prevalence of myopia, it can be estimated that over $22 \%$ of the current world population, that is, 1.5 billion people, are myopic. LASIK has emerged as the refractive corneal surgical procedure of choice for the correction of myopia, with approximately 620,000 to 720,000 procedures performed annually in the last 5 years in the US to correct refractive error. China is one of the countries with the highest incidence of myopia in the world, and increasingly many surgeries will be needed every year to allow people to get rid of their glasses [5] [6] [7].

In conventional optics, the image is differentiated into chromatic and monochromatic aberrations [6]. Monochromatic aberrations can be divided into spherical aberration, coma, astigmatism, curvature of field and distortion. The difference between actual and ideal wavefront in physical optics is the wave front aberration [7], which is an effective strategy for clinically diagnosing and evaluating retinal imaging quality in humans. Retinal imaging quality is affected by various factors, such as pupil size [8], tear film [9] and age [10].

Traditional corneal refractive surgery may correct low order aberrations, such as myopia, hyperopia and astigmatism, but higher-order aberrations, which cause adverse symptoms, including night vision decline, glare, halos, and visual 
discoloration, are not corrected in some patients with surgery [11] [12]. For this reason, individualized corneal refractive surgery guided by wavefront has been performed, and the adverse symptoms mentioned above have been improved. Wavefront-guided individualized corneal refractive surgery has become a hot topic and is regarded as a milestone in the development of corneal refractive surgery [13].

Individualized corneal refractive surgery requires an understanding of the basis of higher-order aberrations before surgery, an examination of the changes in anterior corneal surface and an analysis of their effects on the entire eye system in humans. Therefore, customization of femtosecond laser corneal refractive surgery tailored to the optical properties of an individual is a complex process [14].

In the present study, wavefront aberrations in the anterior corneal surface of the patients were investigated to correct higher-order aberrations with corneal refractive surgery. Our study showed that sphere and coma were the main aberrations in corneal refractive surgery patients. The ratio of coma aberration was increased in the high myopia group, and the sphere was the most dominant type of aberration in the low, moderate and high myopia groups. The frequency of spherical aberration was maintained at approximately $70 \%$ and did not change with the diopter.

Moreover, the changes in diopter and $\mathrm{Q}$ values showed a positive relationship between the low and moderate myopia groups, but there were no significant differences in $\mathrm{Q}$ values between the moderate and high myopia groups. These results indicate that the relationship may not be linear and that the correlation was no longer close when the progress reached a certain degree of myopia. Dubbelman et al. reported that corneal Q values were positively correlated with the increase in myopia degree [15].

The results of the present study showed that the spherical aberrations on the corneal anterior surface increased with increasing myopia degree; however, there was no significance between the aberration types, although the coma aberration was negatively correlated with the degree of myopia. These results suggested that wavefront-guided individualized refractive surgery in a high myopia population can reduce coma aberrations without an obvious advantage in reducing spherical aberrations. This idea requires further verification to remediate the large spherical aberration after corneal refractive surgery in high myopia.

\section{Conclusion}

Overall, wavefront aberration combined with the excimer laser system for individualized corneal refractive surgery has an upstanding theoretical basis. There are some limitations to our study. First, we evaluated only corneal higher-order aberrations before myopic correction refractive surgery, further studies are needed to evaluate the change in the ocular aberrations changes as a function of objective accommodative, relationships between the components of wavefront aberra- 
tions and visual quality and digital correspondence with individualized cutting modes.

\section{Ethics Approval and Consent to Participate}

The institutional review board at the Affiliated Hospital of Yanbian University approved this study, which followed the tenets of the Declaration of Helsinki. Written informed consent was obtained from all patients after the nature and possible consequences of the study were explained.

\section{Availability of Data and Materials}

The datasets and materials used during the current study are available from the corresponding author on reasonable request.

\section{Funding}

This study was supported by the National Natural Science Foundation of China (81560167).

\section{Publisher's Note}

Springer Nature remains neutral with regard to jurisdictional claims in published maps and institutional affiliations.

\section{Conflicts of Interest}

The authors declare no conflicts of interest regarding the publication of this paper.

\section{References}

[1] Durán, J.A., Gutiérrez, E., Atienza, R. and Piñero, D.P. (2017) Vector Analysis of Astigmatic Changes and Optical Quality Outcomes after Wave Front-Guided Laser in Situ Keratomileusis Using a High-Resolution Aberrometer. Journal of Cataract \& Refractive Surgery, 43, 1515-1522. https://doi.org/10.1016/j.jcrs.2017.08.020

[2] Morchen, M., Kaemmerer, M., Mierdel, P. and Seiler, T. (2001) Increased Higher-Order Optical Aberration after Laser Refractive Surgery: A Problem of Subclinial Decentration. Journal of Cataract \& Refractive Surgery, 27, 362-369. https://doi.org/10.1016/S0886-3350(00)00806-3

[3] Moreno-Barriuso, E., Lloves, J.M., Marcos, S., Navarro, R., Llorente, L. and Barbero, S. (2001) Ocular Aberrations before and after Myopic Corneal Refractive Surgery: Lasik-Induced Changes Measured with Laser Ray Tracing. Investigative Ophthalmology \& Visual Science, 42, 1396-1403.

[4] Porter, J., MacRae, S., Yoon, G., Roberts, C., Cox, I.G. and Williams, D.R. (2003) Separate Effects of the Microkeratome Incision and Laser Ablation on the Eye's Wave Aberration. American Journal of Ophthalmology, 136, 327-337. https://doi.org/10.1016/S0002-9394(03)00222-8

[5] Bamashmus, M.A., Hubaish, K., Alawad, M. and Alakhlee, H. (2015) Functional Outcome and Patient Satisfaction after Laser in Situ Keratomileusis for Correction of Myopia and Myopic Astigmatism. Middle East African Journal of Ophthalmolo$g y$, 22, 108-114. https://doi.org/10.4103/0974-9233.148359 
[6] Moshirfar, M., Shah, T.J., Skanchy, D.F., Linn, S.H., Kang, P. and Durrie, D.S. (2017) Comparison and Analysis of FDA Reported Visual Outcomes of the Three Latest Platforms for LASIK: Wavefront Guided Visx iDesign, Topography Guided WaveLight Allegro Contoura, and Topography Guided Nidek EC-5000 CATz. Clinical Ophthalmology, 11, 135-147. https://doi.org/10.2147/OPTH.S115270

[7] Holden, B., Sankaridurg, P., Smith, E., Aller, T., Jong, M. and He, M. (2013) Myopia: An Underrated Global Challenge to Vision: Where the Current Data Takes Us on Myopia Control. Eye, 28, 142-146. https://doi.org/10.1038/eye.2013.256

[8] Osuagwu, U.L., Verkicharla, P., Suheimat, M. and Atchison, D.A. (2018) Peripheral Monochromatic Aberrations in Young Adult Caucasian and East Asian Eyes. $O p$ tometry and Vision Science, 95, 234-238. https://doi.org/10.1097/OPX.0000000000001180

[9] Bruce, A.S. and Catania, L.J. (2014) Clinical Applications of Wavefront Refraction. Optometry and Vision Science, 91, 1278-1286. https://doi.org/10.1097/OPX.0000000000000377

[10] Applegate, R.A., Marsack, J.D. and Sarver, E.J. (2010) Noise in Wavefront Error Measurement from Pupil Center Location Uncertainty. Journal of Refractive Surgery, 26, 796-802. https://doi.org/10.3928/1081597X-20100921-05

[11] Montés-Micó, R., Cerviño, A., Ferrer-Blasco, T., García-Lázaro, S. and Madrid-Costa, D. (2010) The Tear Film and the Optical Quality of the Eye. The Ocular Surface, 8, 185-192. https://doi.org/10.1016/S1542-0124(12)70233-1

[12] Athaide, H.V., Campos, M. and Costa, C. (2009) Study of Ocular Aberrations with Age. Arquivos Brasileiros de Oftalmologia, 72, 617-621.

https://doi.org/10.1590/S0004-27492009000500003

[13] Marcos, S., Barbero, S., Llorente, L. and Merayo-Lloves, J. (2001) Optical Response to LASIK Surgery for Myopia from Total and Corneal Aberration Measurements. Investigative Ophthalmology \& Visual Science, 42, 3349-3356.

[14] Huang, D. and Arif, M. (2002) Spot Size and Quality of Scanning Laser Correction of Higher-Order Wavefront Aberrations. Journal of Cataract \& Refractive Surgery, 28, 407-416. https://doi.org/10.1016/S0886-3350(01)01163-4

[15] Sáles, C.S. and Manche, E.E. (2013) One-Year Outcomes from a Prospective, Randomized, Eye-to-Eye Comparison of Wavefront-Guided and Wavefront-Optimized LASIK in Myopes. Ophthalmology, 120, 2396-2402. https://doi.org/10.1016/j.ophtha.2013.05.010

\section{List of Abbreviations}

SE: spherical equivalent;

RMS: root-mean-square;

SD: standard deviation;

FS-LASIK: femtosecond laser-assisted laser in situ keratomileusis;

ANOVA: analysis of variance;

SA: spherical aberration;

HOAs: higher-order aberrations. 